\title{
Review: Understanding the causes of variation in reproductive wastage among bulls
}

\author{
S. Fair ${ }^{1 \dagger}$ and P. Lonergan ${ }^{2}$ \\ ${ }^{1}$ Laboratory of Animal Reproduction, School of Natural Sciences, Faculty of Science and Engineering, University of Limerick, Limerick P61 C996, Ireland; ${ }^{2}$ School of \\ Agriculture and Food Science, University College Dublin, Belfield, Dublin 4 D04 N2E5, Ireland
}

(Received 5 February 2018; Accepted 27 March 2018; First published online 21 May 2018)

The ability to predict the fertility of bulls before semen is released into the field has been a long-term objective of the animal breeding industry. However, the recent shift in the dairy industry towards the intensive use of young genomically selected bulls has increased its urgency. Such bulls, which are often in the highest demand, are frequently only used intensively for one season and consequently there is limited time to track their field fertility. A more pressing issue is that they produce fewer sperm per ejaculate than mature bulls and therefore there is a need to reduce the sperm number per straw to the minimum required without a concomitant reduction in fertility. However, as individual bulls vary in the minimum number of sperm required to achieve their maximum fertility, this cannot be currently achieved without extensive field-testing. Although an in vitro semen quality test, or combination of tests, which can accurately and consistently determine a bull's fertility and the optimum sperm number required represent the 'holy grail' in terms of semen assessment, this has not been achieved to date. Understanding the underlying causes of variation in bull fertility is a key prerequisite to achieving this goal. In this review, we consider the reliability of sire conception rate estimates and then consider where along the pregnancy establishment axis the variation in reproductive loss between bulls occurs. We discuss the aetiology of these deficiencies in sperm function and propose avenues for future investigation.

Keywords: sperm, semen, bovine, prediction, fertility

\section{Implications}

A substantial number of bulls whose semen passes the postthaw quality control checks in artificial insemination (AI) centres have reduced fertility in the field. Although this is undoubtedly multifactorial, the purpose of this review is to attempt to identify where in the sequence of events, sperm from low-fertility bulls compromise the establishment of pregnancy. Understanding this will aid in the development of improved strategies for the early detection of bull subfertility and/or its amelioration.

\section{Introduction}

Animal breeding centres have for years relied upon classical microscopy-based techniques to assess sperm motility (total and progressive) and morphological parameters as part of their quality control programmes. However, work by our group and others have demonstrated that bulls whose semen passes these minimum post-thaw quality control checks at

${ }^{\dagger}$ E-mail: sean.fair@ul.ie an Al centre can still vary in their field fertility. Traditional progeny testing schemes allowed semen from individual bulls to be released over a prolonged period and once nonreturn rate data became available, semen from sub-fertile bulls could be taken off the market. To protect against the risk of reduced fertility, Al companies typically utilise excessive sperm numbers in each straw (15 to 20 million). A number of studies with frozen-thawed conventional semen has revealed that most Holstein sires used in $\mathrm{Al}$ achieve their individual maximum pregnancy rate value at 2.5 to 5.0 million total sperm per dose, with a range from 0.5 to 12 million sperm per dose (Den Daas et al., 1998). Although the blanket approach of increasing the sperm number in all bulls guards against individual bulls with compensable sperm defects, this approach limits the number of straws that can be processed per ejaculate, thereby limiting supply of their semen.

With the advent of genomic selection, semen is now being collected from bulls at a younger age and these elite bulls are typically only used intensively for one season as they are then surpassed by the next generation of genetically superior bulls. This intensive use and high rate of Al sire turnover leaves insufficient time to adequately assess the fertility 
status of a bull before wide-scale use of his semen in the field, especially in seasonal grass-based production systems, such as those operated in Ireland and New Zealand. In these pasture-based systems, the breeding season is condensed into $\sim 3$ months so as to calve cows compactly at the start of the grass-growing season. These young bulls also produce fewer sperm per ejaculate, and therefore the luxury of putting excessive numbers of sperm in a semen straw for bulls where demand for semen far exceeds supply is costly for the Al centre and limits farmer access to such elite bulls. Therefore, a reliable in vitro test or a combination of tests, which could accurately predict the outcome of insemination would facilitate the identification of sub-fertile bulls before their widespread use in the field and the more efficient use of the semen of high fertility bulls through the reduction of sperm number per straw.

There are numerous recent studies and comprehensive reviews on the prediction of bull fertility (Sellem et al., 2015; Utt, 2016; Abdollahi-Arpanahi et al., 2017) and we do not propose to replicate these here. Instead, this review will focus on the caveats surrounding sire fertility estimates and on the specific reasons why bulls with apparently normal semen vary in their fertility. We assess the usefulness of in vitro assessments to mimic the in vivo events leading up to the establishment of a pregnancy. Finally, we propose likely avenues for fruitful future investigation.

\section{Sire fertility estimates; establishing a reliable phenotype}

Many studies that use in vitro approaches to investigate bull fertility fail to understand the limitations of even the best designed sire fertility estimates and thus many studies are flawed from the start due to an unreliable fertility phenotype. To accurately rank sires, a detailed understanding of factors affecting the models are required. Most Al centres worldwide track bull fertility using either non-return rates or more accurate (and complex) adjusted sire conception rate (SCR) models that account for environmental factors (herd, technician, month of insemination, age of cow, cow genotype, days in milk, milk production, etc.) and express a bull's fertility relative to a population mean of 0\%. A detailed review by Amann and DeJarnette (2012) demonstrated that the fertility of $90 \%$ of the bulls marketed is within \pm 3 percentage points of the mean of the bull population. This is consistent with our preliminary data on Irish Al bulls. On a population basis, Amann and DeJarnette (2012) concluded that Al companies will never be able to measure 'fertility' more precisely than \pm 3 percentage units from the population mean because of the difficulty in controlling many factors including: binomial variation, herd environment, measurement errors, and bias in semen use. Another key attribute in understanding 'Sire Fertility' is the number of inseminations required per sire to confidently rank them on their fertility. The same study illustrated that to confidently (two tailed test, $P=0.05,80 \%$ power) differentiate sires $\pm 4 \%$ from the average of the bull population each sire must have a minimum of 1000 inseminations. With just 300 inseminations, as is often the case in studies attempting to predict the fertility of an individual ejaculate, it is only possible to confidently differentiate sires $\pm 7 \%$ from the average. Thus, failure to recognise limitations in any estimate of potential fertility leads to over interpretation of small differences among sires in apparent fertility.

\section{Do artificial insemination bulls rank the same when used under different conditions?}

Although the timing of insemination relative to onset of oestrus does not influence the fertility of above average fertility sires, a significant drop in fertility was reported when semen from below average sires was inseminated in early and mid-oestrus (Macmillan and Curnow, 1977), suggesting differences in the fertile lifespan of sperm in the female tract. Despite this fact, once a day Al is now widely used with similar fertility achieved to when twice a day Al is performed, irrespective of whether fresh or frozen-thawed semen is used (Xu, 2017).

Optimum fertility can be achieved with a much lower sperm number when fresh (liquid) rather than frozen-thawed semen is used ( 2 to 5 million v. 15 to 20 million sperm, respectively; Murphy et al., 2017), maximising the utilisation of genetically superior sires. The higher sperm numbers in cryopreserved semen compensate for the damage during the freeze-thaw process compared with fresh semen and, on average, the same level of fertility is achieved with both types of semen (Murphy et al., 2015). In a data set analysed by our group, bulls which have low fertility with frozenthawed semen tend to have low fertility with fresh semen although there are some exceptions (Figure 1). Of the 16 bulls used across 66252 inseminations, nine bulls varied substantially in the fertility achieved between fresh and frozen-thawed semen (five bulls were higher with fresh semen and four were higher with frozen-thawed). In contrast, Vishwanath and Shannon (2000) reported that bulls

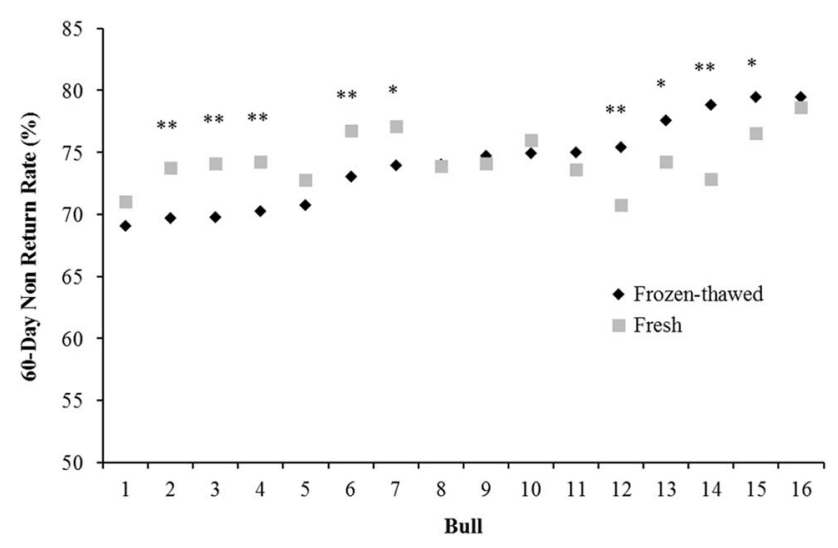

Figure 1 Characterisation of the variation between 60 day non-return rate in 16 bulls with split ejaculates used as fresh or frozen-thawed semen ( $n=66252$ inseminations). ${ }^{*} P<0.05,{ }^{*} P<0.01$ between semen type within bull. 
generally followed the same fertility trend with fresh and frozen-thawed semen when optimum sperm numbers were used (2.5 million/dose for fresh and 20 million/dose for frozen-thawed) but when suboptimum sperm numbers were used ( 0.5 million/dose for fresh and 5 million/dose for frozenthawed) fertility declined by $7 \%$ and $7.9 \%$ for fresh and frozen-thawed, respectively. More importantly, there was a significant bull by sperm number interaction, whereby some bulls dropped by over $20 \%$ when lower sperm numbers of frozen-thawed semen was inseminated and some bulls performed the same as with 20 million sperm. This trend of a greater variation among bulls at lower sperm concentrations was also observed by Den Daas et al. (1998) where maximum fertility for individual bulls was achieved at differing sperm concentrations, and the sperm numbers needed to obtain $95 \%$ of the maximal conception rate ranged from 1 to 11 million sperm per dose. This highlights the variability in the susceptibility of an individual bull's semen to the freeze-thaw process and how freezing protocols should be customised to individual bulls, an area of research that has received little attention in recent years. It also illustrates that individual bulls have different maximum fertility and the number of sperm required to achieve this varies among bulls.

In order to minimise differences in pregnancy rates among individual sires, over-compensation of sperm numbers typically occurs in the preparation of frozen-thawed semen, resulting in a sperm concentration that considerably exceeds the number of sperm necessary for maximum fertility. Thus, the 'true fertility' potential of a bull in the field is masked by the greater sperm number per insemination dose, and this needs to be considered when attempting to understand the variation in SCR using in vitro assays. For example, consider an $\mathrm{Al}$ centre which processes semen at 15 million sperm per inseminate (as is typical) with an overall mean calving rate across all its bulls of $53 \%$. A comparison of two of their bulls with a calving rate of $60 \%$ would lead to the conclusion that both bulls were of 'high fertility' and in any retrospective 'prediction type analysis', they would be treated as such. However, now consider that if assessed at a lower sperm number, one bull would have had the same fertility at a dose of 5 million sperm per straw while the second bull would have required 12 million sperm for this level of fertility. As the only fertility data available to the Al centre was at a concentration of 15 million sperm, both bulls would be considered to have the same fertility phenotype yet there are distinctive differences in the ability of their sperm to establish a pregnancy after the freeze-thaw process. Therefore, fertility can only be actually determined under conditions where sperm numbers are limiting (Hammerstedt, 1996) and it is not surprising that there is difficulty in identifying the causes of bull subfertility when a dubious fertility phenotype is used at the start. Ideally, the number of sperm at which a bull's fertility reaches a plateau should be determined, but tracking semen straws with varying sperm concentrations in the field poses major logistical issues for most Al companies.

\section{Where does reproductive wastage occur in bulls that vary in their sire conception rate following artificial insemination?}

There have been a plethora of publications on the prediction of sire fertility using sperm functional (Sellem et al., 2015), molecular (Rahman et al., 2017) and genomic (Puglisi et al., 2016) models as well as combinations of these. Despite this, there is still no single test, or combination of tests, which can reliably predict bull fertility. Very few studies have focused on attempting to understand why bulls whose semen has normal post-thaw motility and morphology, as viewed under a microscope, can still vary in fertility by up to $20 \%$ points. For most commercial situations, fertility is defined as cows either failing to return to oestrus (non-return rate) or confirmed pregnant by means of ultrasound scan, rectal palpation, blood progesterone or a calving event. These estimates of pregnancy status following insemination are incapable of differentiating the reasons for pregnancy failure. What is clear is that semen is deposited into the uterine body and the chance of pregnancy varies among bulls. The possible reasons for this are presented in Figure 2 and the associated published studies are then discussed.

\section{Sperm proteome and its relationship to the establishment of pregnancy}

During ejaculation, sperm becomes coated in proteins immediately during ejaculation that is secreted from the epididymides as well as the accessory glands and even though bull semen is typically diluted 15 to 25 fold during semen processing, the effects of seminal plasma proteins are likely to be maintained as they adhere to sperm rapidly upon ejaculation. Numerous studies have focused on characterising the proteomic composition of the seminal fluid (which also contains epididymal fluid) across a range of species (Druart et al., 2013) and related these to fertility. Some of the seminal plasma proteins that have been positively related to bull fertility include osteopontin $\left(\mathrm{Ca}^{2+}\right.$-binding protein) and lipocalin-type prostaglandin D synthase (Cancel et al., 1997), telomeres-1 protein (POT1) (Aslam et al., 2014) while other seminal plasma proteins have been negatively correlated to fertility including prostaglandin E2 receptor EP3 (PTGER3) (Aslam et al., 2014). More functional studies are required to validate these and to characterise how exactly they influence the establishment of pregnancy.

Other studies have mapped the proteome of bull sperm and have reported correlations between specific proteins and sperm motility, morphology as well as fertility ( $D^{\prime}$ Amours et al., 2010). Sperm proteins can be broadly categorised into energy-related, structural and other functional proteins and sperm-bound proteins from bulls of varying fertility have been related to spermiation and energy homoeostasis, membrane function, sperm-egg interactions and cell cycle regulation as well as glycolysis, post-translational changes during sperm maturation, capacitation and protection against oxidative stress, to name but a few (Gaviraghi et al., 2010; Park et al., 2012). Molecular defects in some of these 


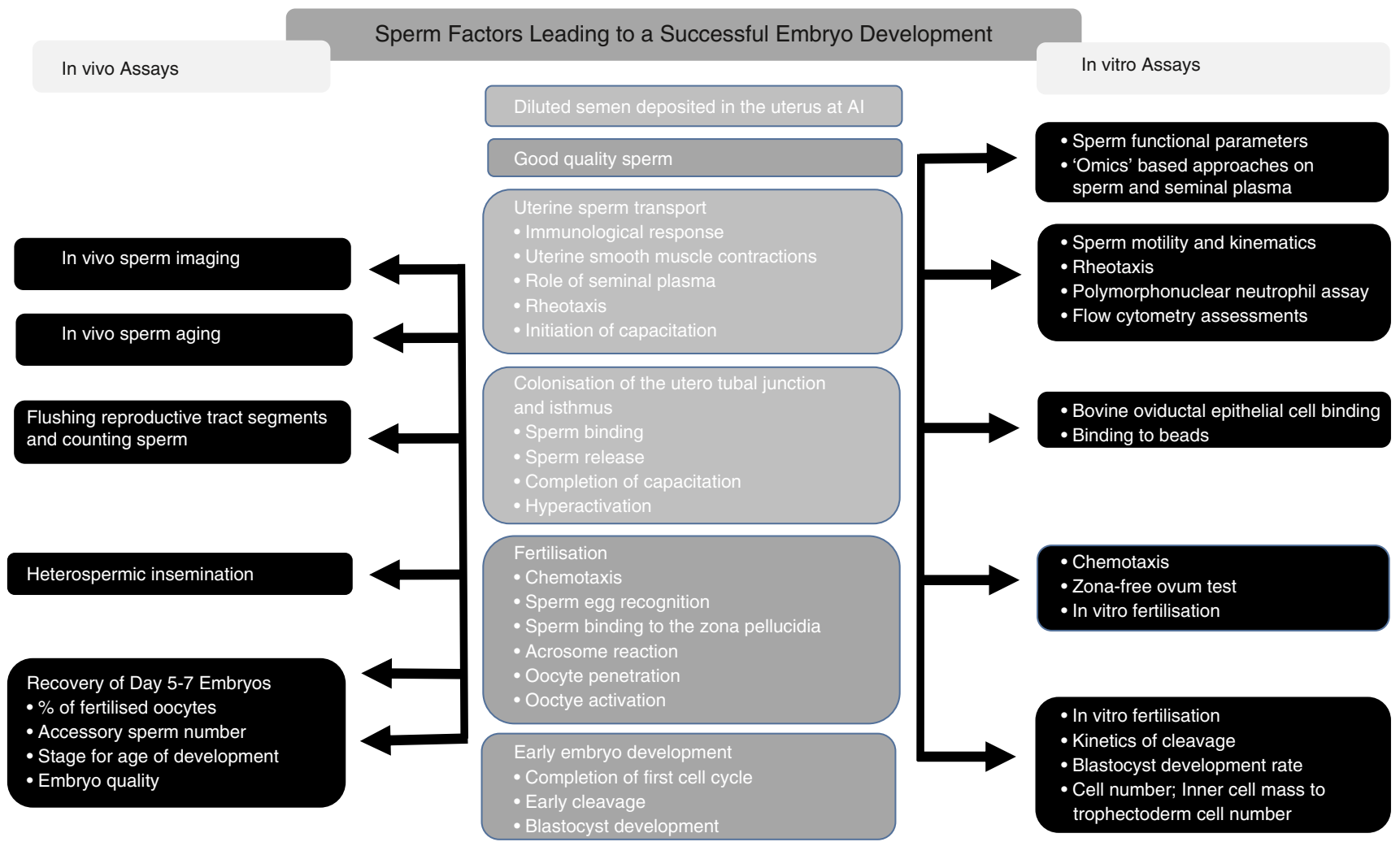

Figure 2 Events leading to the establishment of a viable embryo following artificial insemination (Al; centre column), with the in vivo (left column) as well as the in vitro (right column) assessments that have been used in published studies to characterise the differences in these events between bulls of varying fertility.

proteins have been reported to be associated with low fertility or in certain cases, infertility. Somashekar et al. (2017) investigating sperm proteomic signatures regulating sperm function and fertility reported calmodulin (CALM1), spermadhesinZ13 (SPADH2), and phosphatidylethanolaminebinding protein 4 (PEBP4) to be present in higher amounts on the sperm of high fertility bulls with PEBP4 being absent in infertile bulls. An earlier study by the same group reported that the seminal plasma protein PDC-109 was more abundant on sperm from low-fertility bulls (Somashekar et al., 2015). The exact role of many of these proteins in bull fertility is still unclear, and thus the current challenge for reproductive biologists is to move from lists of identified proteins to an informed understanding of biological function given that they control key physiological events in the female tract.

Sperm communication and interaction with the female tract The immunological responses to sperm and seminal plasma in the female tract are of considerable interest as these processes influence sperm capacitation, transport, selection, fertilisation as well as early embryo development (Schuberth et al., 2008). The local immune responses of the epithelial lining, regulated by its secretions, constitute the main part of the mucosal innate immunity inside the uterus and oviduct which is largely mediated by cytokines, chemokines, and prostaglandins (Bulek et al., 2010). Much of the focus in humans and rodents have been on the bioactive signalling agents in seminal plasma and how they evoke gene expression and cellular changes in the innate immune system (see review by Schjenken and Robertson, 2014). The presence of sperm, seminal plasma and semen diluent causes a triggering of the first line of defense against foreign cells through increased production of pro-inflammatory cytokines, leading to an influx of polymorphonuclear neutrophils (PMNs) into the lumen of the female tract (Marey et al., 2014). Polymorphonuclear neutrophils have been reported to clear dead and immotile sperm, but also motile sperm (Li and Funahashi, 2010) and the presence of activated phagocytes can lead to decreases in sperm motility due to increased production of reactive oxygen species (ROS). Oxidative stress is known to be a major factor regulating the vitality and functionality of sperm; however, the precise implications of increased ROS within the female tract are not well understood. Sperm from bulls with below average fertility had significantly greater ROS production compared with above average fertility bulls (Kumaresan et al., 2017) which has in turn been related to increased deleterious effects of lipid peroxidation on the membrane and DNA integrity (Koppers et al., 2011).

Using an in vitro model, Marey et al. (2016) demonstrated that endothelin-1 may be involved in supporting bull sperm survival until fertilisation through the protection of sperm from phagocytosis by PMNs in the bovine oviduct. In the macaque, beta-defensin 126 (BD126) has been reported to protect sperm from immune-recognition and binding of anti-sperm antibodies (ASA; Yudin et al., 2005). Anti-sperm 
antibodies are produced in response to antigens present on sperm and can account for reduced sperm viability but also higher sperm mortality in the female reproductive tract (Rossato et al., 2004). It is estimated that ASA are responsible for as much as $40 \%$ of unexplained fertility cases in humans and recent studies have also revealed a high level of ASA in both serum and seminal plasma from bulls that have a negative effect on their fertility through the prevention of capacitation (Zodinsanga et al., 2015).

$\beta$-defensin glycoproteins coat sperm and have been identified as having a role in modulating the inflammatory response to enhance sperm survival (Yudin et al., 2005). Although the function of $\beta$-defensins in reproduction have not, until recently, been explored in farm animal species, knockout of a $\beta$-defensin gene cluster in male mice resulted in complete sterility (Zhou et al., 2013). In men, variation in the $b d 126$ sequence contributes to subfertility (Tollner et al., 2011) while the BD126 peptide has been reported to mediate sperm binding to the oviductal epithelium (Tollner et al., 2008). Our group has reported bovine beta-defensin 126 (BBD126) to be extensively expressed in the reproductive tract of the bull with preferential protein expression in the cauda epididymis (Narciandi et al., 2011) and on sperm (Narciandi et al., 2016) with similar binding patterns on the sperm surface to macaque. Beta-defensin 126 increases the net negative charge on sperm (Tollner et al., 2012), increases sperm motility, mucus penetration in vitro (FernandezFuertes et al., 2016) as well as sperm binding to oviductal epithelium in vitro (Lyons et al., 2018). We have also recently characterised the genetic variation in bovine $\beta$-defensin genes as well as completing the first whole-exome sequencing of Al bulls of divergent fertility (Whiston et al., 2017). This dual approach successfully identified novel variants in both beta-defensin and FOXJ3 genes as potentially regulating SCR through differential oviductal binding ability, as assessed in vitro (Whiston et al., 2017). Using a microarray-based approach, Legare et al. (2017) characterised the expression of genes along the caput, corpus and cauda epididymis in bulls which differed in SCR. The transcriptional profiles between subfertile and fertile bulls clustered most closely in the cauda and corpus segments, whereas the profiles in the caput segment were distinct between sub-fertile and fertile bulls. Of the differently expressed genes, 10 were related to reproductive function and five were associated with the defense response (of which two belonged to the defensin family, namely DEFB119, DEFB124). Bulls carrying mutations in genes which encode these immunoregulatory peptides could produce sperm of higher immunogenicity which could well contribute to reduced sperm survival in the female reproductive tract and subfertility.

During ejaculation, a binder of sperm proteins (BSPs) are secreted by bovine seminal vesicles into seminal plasma and immediately absorbed onto sperm (Leahy and de Graaf, 2012). Of these, BSP1, BSP3, BSP5 have been reported to facilitate uncapactitated bull sperm in binding to the epithelial lining of the utero-tubular junction and isthmus, forming a sperm storage reservoir (Hung and Suarez, 2012).
However, during the completion of capacitation, changes in their composition on sperm play a role in releasing sperm from these storage reservoirs. The ability of sperm to bind to the oviductal epithelium appears critical to establishing a viable sperm population in the oviducts and may aid in overcoming any asynchrony between the timing of $\mathrm{Al}$ and ovulation. A number of studies have investigated the interaction between sperm and the oviductal epithelium in vitro and demonstrated that it is mediated by fucose (Lefebvre et al., 1997). Previous in vivo work has reported that the timing of insemination is more important for low and average fertility bulls compared with high fertility bulls (Macmillan and Watson, 1975) suggesting that there is a reduced ability of sperm from low-fertility bulls to develop a reservoir of functional sperm at the utero-tubular junction and in the oviducts. Interestingly, Yousef et al. (2016) reported that bovine oviductal epithelial cells provide an anti-inflammatory environment and the sperm-epithelial binding further strengthens this, leading to the suppression of PMNs in the bovine oviduct. In addition to facilitating sperm binding, Lessard et al. (2011) investigating the aetiology of idiopathic infertility in a beef bull established that his sperm were unable to undergo the acrosome reaction, when induced using calcium ionophore, and related this to the level of BSP1 that was much greater on sperm from the infertile bull compared with that of his sire.

It is clear that there is cross-talk between semen and the female tract, starting in the uterus with the induction of an inflammatory response and continuing in the oviduct through sperm binding and subsequent release. There is evidence that bulls vary in their capacity to complete these physiological processes and indications are that this is related to the surface proteome of sperm that is influenced by both the epididymal secretome and the composition of seminal plasma. The focus, therefore, should be on characterising these parameters from bulls of divergent fertility with a view to identifying key biomarkers (not just proteins), which can then be used in functional studies to better understand how these regulate the dialogue between sperm and female reproductive tract.

\section{Sperm transport in the female tract}

In vivo assessments, either by flushing sperm from the segments of the reproductive tract following Al or using confocal imaging of sperm in the female tract as has been performed in sheep (Druart et al., 2009), are unlikely to be sensitive enough to detect differences among bulls with varying SCR. Other approaches such as mucus penetration tests, assessment of accessory sperm number following $\mathrm{Al}$ and heterospermic insemination have been used to understand why some males sperm may be better able to navigate the female reproductive tract and its secretions than others.

The use of mucus penetration assays in vitro, which assess the ability of the sperm to travel through a capillary filled with artificial mucus or cervical mucus from oestrus cows has been used as a proxy for assessment of sperm transport and has been correlated to SCR (Al Naib et al., 2011). 
Although these studies were conducted in a static mucus environment, the development of microfluidic systems enables the characterisation of sperm rheotaxis, a phenomenon whereby sperm swim against a flow (Miki and Clapham, 2013) and offers a way of assessing sperm migration ability. Although chemotaxis may guide sperm towards the ovulated oocyte once it is in its vicinity in the ampulla, rheotaxis has been proposed as a long-range guidance cue for sperm navigation along the female tract. Rheotaxis requires rotation of the sperm, which requires CatSper calcium-selective ion channels. CatSper glycoproteins form the sperm-specific voltage-gated $\mathrm{Ca}^{2+}$ channels localised along the membrane of the sperm flagellum. CatSper channels contain glycoproteins that are involved in positioning regulation and recent work by our group has demonstrated that hyperactive bull sperm exhibit an increased rheotaxis response (Johnson et al., 2017). Targeted disruption of CATSPER 1, CATSPER2, CATSPER3 or CATSPER4 inhibits hyperactivated motility and thus rheotactic reponse (Johnson et al., 2017). There are no published studies on the rheotactic response of bulls differing in SCR. The advent of 3-D printing will no doubt facilitate the development of more physiological and sensitive models for studying this as well as sperm interaction with the female tract and its secretions.

Heterospermic insemination involves the insemination of the semen mixture at the one point in time into the same female and thus levels the playing field. Each sperm should have an equal chance to reach and fertilise the oocyte without influence by technician, cow age/parity/genetic merit/days in milk, timing of insemination relative to oestrus, season and management (Beatty et al., 1969). For this reason, it has been reported that heterospermic insemination is up to 170 times more sensitive in ranking reproductive outcome than homospermic measures (Flint et al., 2003). To put this into context, homospermic insemination requires thousands of inseminations to compare fertility of two males accurately while heterospermic insemination has been reported to be able to test the fertility of a bull accurately and rapidly using fewer than 100 females. Overstreet and Adams (1971) inseminated a mixture of equal numbers of labelled and unlabelled rabbit sperm from two bucks and flushed the reproductive tract of does 6 or $13 \mathrm{~h}$ later for evidence of selective transport and sperm viability. The numbers of sperm from each male in each of the segments of the reproductive tract were equal at $6 \mathrm{~h}$, but by $13 \mathrm{~h}$ sperm from the superior buck predominated in the uterus and oviducts. More sperm from the superior buck were attached to the zona pellucida and fertilised more oocytes. When semen was placed in the oviducts the sperm were present in equal numbers in the vicinity of the oocyte but the skewed proportion of offspring and labelled sperm penetrating the oocyte still favoured the superior buck. Using heterospermic insemination of fluorescently labelled sperm, Ferreira (1972) reported that sperm number recovered from the vagina, uterus and oviduct was similar among males, as was the number of sperm bound to the zona pellucida of recovered oocytes. These observations lead the authors of these aforementioned studies to conclude that sperm are present in equal numbers in the immediate vicinity of the oocyte and perhaps rate of oocyte penetration or subsequent activation of the oocyte differed among males. This is also in agreement with Macmillan and Watson (1975) who reported that all bulls have a similar opportunity to fertilise when Al occurred close to ovulation, but when Al occurred at longer intervals before ovulation, the sperm of some bulls, which were obviously present at longer intervals to $\mathrm{Al}$, were no longer alive or capable of fertilising an oocyte. These studies emphasise the importance of having a population of functional sperm in the oviducts at the time of ovulation.

\section{Fertilisation and early embryo development}

Fertilisation success following $\mathrm{Al}$ in cattle with semen from high fertility sires is in the order of $90 \%$ to $95 \%$ in heifers and moderate yielding cows (Diskin and Sreenan, 1980). A meta-analysis by Sartori et al. (2010) estimated that fertilisation rates in North American high-producing Holstein cows to be $83 \%$ while pregnancy rates of similar genetic merit lactating cows to be $33 \%$. Several studies have been performed over the last 30 years in which cows have been slaughtered at various time-points post insemination in order to assess embryo viability. The majority of this reproductive wastage in single-ovulating cows had been attributed to early embryo loss with $<50 \%$ of recovered embryos from high yielding lactating cows viable 7 days after Al followed by additional losses through Day 34 (Sreenan and Diskin, 1986). Sartori et al. (2002) demonstrated that lactating cows had poorer quality Day 5 embryos than both heifers and dry cows but surprisingly more accessory sperm indicating that delayed sperm transport was not a causative effect. In singleovulating cows, most embryos and $\sim 80 \%$ of unfertilised oocytes had at least one accessory spermatozoon (Cerri et al., 2009) while Sartori et al. (2002) reported mean values of 18-42 sperm in embryos and 18 in unfertilised ova. All of these aforementioned studies were focused on cow factors and there is a complete dearth of published studies focusing on the relationship between SCR and the contribution of the sperm to failure of sperm transport, fertilisation or embryo development. Using a small number of bulls with below and above average fertility, Ortega et al. (2017) recently assessed the contribution SCR to pregnancy establishment and reported that bulls with a higher SCR had an advantage in terms of in vivo and in vitro production of embryos. In the same study there was no effect of SCR on preimplantation conceptus elongation and development. Kumaresan et al. (2017) reported that bulls with below average fertility had a significantly lower sperm population with intact acrosomes post-thawing compared with bulls with above average fertility, similar to earlier reports (Singh et al., 2016).

In vitro fertilisation (IVF) is a powerful tool to assess the fertilising ability of sperm. The kinetics of sperm penetration (Ward et al., 2002) as well as the first cell cycle (Comizzoli et al., 2000) and of the first mitotic cleavage after fertilisation (Lonergan et al., 1999) are highly correlated with the 
likelihood of an embryo developing to the blastocyst stage and to the quality of those embryos (Dinnyes et al., 1999). Ward et al. (2001) was able to discriminate between bulls of high and low field fertility based on the timing of the first cleavage division post insemination in vitro, whereby embryos fertilised from high fertility bulls cleaved first and significantly more of these early cleaving zygotes were more competent in terms of development to the blastocyst stage than those that cleaved later. The same study reported a significant correlation between Day 7 blastocyst yield and field fertility while a separate study reported an effect of SCR and cleavage rate (Al Naib et al., 2011). In contrast, Kropp et al. (2017) reported no differences in the morphology and development to the blastocyst stage but preimplantation embryos derived from high and low-fertility bulls displayed significant transcriptomic differences, which they postulated could influence the reprogramming of the early embryo. Therefore, the evidence suggests that a portion (contribution will vary among sires) of the embryo death before $\sim$ Day 8 is caused by the fertilising sperm, but the specific aspect of the sperm causing this effect is unclear.

\section{Role of sperm DNA integrity and methylation signature} Individual bulls vary in the levels of sperm DNA fragmentation that they exhibit (Takeda et al., 2015) and there appears to be a growing link between this parameter and early embryonic loss and even foetal development and health of the offspring (Evenson and Jost, 2000). During spermiogenesis, sperm chromatin is remodelled whereby core histones are replaced by transition proteins which are subsequently replaced by protamines resulting in chromatin that is tightly compacted and resistant to denaturation (Filho et al., 2015). This compaction is necessary to protect sperm chromatin during transit through the epididymis and female reproductive tract. Shortly after fertilisation, sperm protamines are replaced by maternal histone variants. Thus, defects of sperm chromatin structure affect sperm function during fertilisation, first cleavage and early embryonic development. Inadequate sperm chromatin protamination and DNA integrity were associated with defects in bull sperm chromatin condensation, coinciding with reduced in vivo fertility (Dogan et al., 2015). Disruption to defective chromatin packaging during spermiogenesis results in sperm that are susceptible to denaturation and there is growing evidence, that the status of sperm chromatin at the time of fertilisation can influence embryonic survival (Sakkas et al., 2002).

A number of studies have demonstrated a relationship between the levels of DNA fragmentation in bull sperm and SCR (Kumaresan et al., 2017) with a high level of DNA fragmentation correlated to sperm morphology (Nagy et al., 2013) as well as to a reduced sperm fertilisation potential. DNA fragmentation values of between $7 \%$ and $10 \%$ have been reported to be indicative of low Al success in bulls as DNA damage can jeopardise embryonic development (Karoui et al., 2012). However, it must also be noted that many bulls that have lower fertility do not always exhibit increased levels of DNA fragmentation as Rodriguez-Martinez and Barth (2007) reported no direct correlation of DNA fragmentation with fertility. Therefore, like many other in vitro parameters, DNA fragmentation seems more useful when using the negative biomarker approach whereby high levels indicate sperm defects, but low levels do not guarantee fertility.

It is has been known for some time that, at the time of fertilisation, sperm deliver much more than just DNA, but rather an entire package including RNAs, transcription factors, and cell signalling molecules (Krawetz, 2005). Although a number of studies have demonstrated that the transcriptome is significantly different among sires of varying fertility (Feugang et al., 2010), it has only recently been reported that the embryonic transcriptome is influenced by the 'RNA package' delivered by sires of varying fertility status at the time of fertilisation (Kropp et al., 2017). The same study characterised the epigenetic signature of the sperm between bulls of high and low fertility and revealed 76 regions to be differentially methylated between sires of divergent fertility. Although cleavage and blastocyst rate was not affected, the resultant IVF-derived embryos had significantly different transcriptomic profiles with genes relating to metabolic processes and catalytic activities more highly expressed in sperm from high fertility bulls. Errors relating to the condensation of the DNA during spermatogenesis as well as maintenance of epigenetic marks could possibly explain the differences in embryonic gene expression. Indeed, lower levels of DNA condensation, protamine exchange, and higher DNA damage have been observed in sperm from lower fertility bulls in comparison to higher fertility bulls (Dogan et al., 2015). In addition, a recent study focusing on the epigenetic profiles of young bulls highlighted that 10-month-old bulls have a different sperm DNA methylation pattern compared with both 12- and 16-monthold bulls (Lambert et al., 2018). Given the current trend of using semen from elite genomically selected bulls, this study demonstrates that such bulls not only have poorer sperm motility and morphology but also an altered epigenetic profile that has the potential to influence embryonic development as well as the genotype and the phenotype of the subsequent offspring.

\section{Sperm RNA and its relationship to sire conception rate}

Ejaculated sperm are 'stripped-down' cells, equipped with a strong flagellum to drive them through an aqueous mucus environment but unencumbered by cytoplasmic organelles. As a result they are transcriptionally inactive but do retain remnant messenger RNA (mRNA) that are left over from spermatogenesis that can be used for diagnostic purposes. Thus, transcripts (and translation) products of genes are present even in functionally mature ejaculated sperm but are products of later spermatids (or earlier) active gene expression, processes that cease before spermiation. Recent analyses are challenging this belief suggesting that the rich repertoire of coding and non-coding RNAs in sperm is not a haphazard remnant from spermatogenesis in the testes but a carefully selectively retained and functionally coherent collection of RNAs (Das et al., 2013). More recent 
interpretations suggest human sperm retain mRNA that can be translated into protein in the oocyte after fertilisation (Jodar et al., 2013). However, their precise role in the regulation of fertilisation and early embryonic development in the bovine remains to be determined. The mRNA expression of proteins associated with sperm function in bulls of high and low SCR reported a number of genes correlated with fertility status (Kasimanickam et al., 2012). Feugang et al. (2010) analysed the RNA profiles of sperm from high and low-fertility Holstein bulls using Affymetrix bovine genechips and reported differential expression in the abundance of mRNAs. A total of 415 transcripts out of 24 000 were differentially detected in sperm collected from both fertility groups. Sperm from the low-fertility bulls were deficient of transcripts for transcriptional and translational factors while sperm from high fertility bulls contained higher concentrations of transcripts for extracellular space and membrane protein locations.

Short non-coding microRNAs (miRNAs) do not code for proteins, but various studies have reported that miRNAs regulate gene expression and also play a major role in embryo development (Boerke et al., 2007). However, their precise role in the regulation of fertilisation and early embryonic development in the bovine remains to be determined. miRNA profiling from high and low-fertility bulls has also been previously performed (Govindaraju et al., 2012) with seven miRNAs (aga-3155, -8197, -6727, -11796, $-14189,-6125,-13659)$ being differentially expressed. Tscherner et al. (2014) reported that the miR-34 miRNAs play a role in developing bovine gametes and suggested that individual variation in sperm miR-34 family abundance may be a biomarker of male bovine fertility. In addition, single nucleotide polymorphisms in target mRNA or miRNA have revealed associations with traits of economic interest and highlight the potential use of miRNAs in future genomic selection programs (Fatima and Morris, 2013). Sperm miRNA may be useful in understanding the transmission of epigenetic characteristics to male calves and its connection with the transgenerational inheritance of fertility/subfertility related traits. High-throughput RNA sequencing approaches will aid in the determination of the key coding and noncoding transcripts controlling sperm function and thus SCR.

\section{Future directions of research directed at understanding the aetiology of idiopathic bull fertility}

Male fertility has received far less attention in comparison to female fertility yet it is undoubtedly complex and definitely multifactorial. Despite many positive findings, the small numbers of bulls and, in some cases, an unreliable fertility phenotype due to insufficient insemination records for individual bulls as well as issues around sperm number used make interpretation of the findings of many studies challenging and sometimes unrepeatable when applied to different datasets. Despite this, it is now clear that that the sperm deliver not only DNA but also RNA and signalling factors to the oocyte at fertilisation. The most fruitful avenues of further investigation would appear to be around the differences among bulls in the kinetics of sperm penetration as well as completion of the first cell cycle and of the first mitotic cleavage after fertilisation. Embryos that cleave first are most likely to successfully reach the blastocyst stage and the quality of these embryos is superior at the preimplantation stage than later developing embryos. The pathophysiology of delayed cleavage may reside with the non-coding RNAs and or alterations in epigenetic signatures within the sperm which are most likely to be altered during testicular development or by epididymal modifications. An in-depth examination of these factors may shed new light on the cross-talk between bovine sperm and the early stages of embryo development; and importantly how this may be perturbed in bulls of low fertility. Future studies will no doubt take advantage of recent advances in high-throughput techniques to study DNA, RNAs, proteins, lipids, glycans and metabolites in combination. These 'OMICS'-based technologies have increased our capacity to study new and novel aspects of sperm function and to get a broader view of these complex biological systems. They hold the main advantage of providing large volumes of information at relatively low cost and recent advances in bioinformatics enable the analysis and interpretation of large datasets in a more integrated systems biology approach.

Like so many studies thus far, these technologies will undoubtedly produce lists of biomarkers that are different between bulls of varying fertility. The major challenge then is to define which ones are physiologically important. For this, we need novel functional approaches comprising of both in vitro and in vivo methods. However, as outlined earlier in this review, before we go down this path we must be cognisant of the limitations of sire fertility estimates especially when inseminations are performed with high numbers of sperm. Then, we should ensure experiments are sufficiently powered with bulls across a wide range of the fertility spectrum in the quest to identify the reasons for the variation in SCR.

\section{Acknowledgements}

The authors acknowledge the contribution of Ms Edel Murphy in the compilation of this manuscript. The authors are supported by funding from Science Foundation Ireland (grant no. 16/IA/4474).

\section{Declaration of interest}

The authors have no vested interests to declare.

\section{Ethics statement}

This manuscript is a review of existing published work and as such did not use animals for its' preparation.

\section{Software and data repository resources}

None.

\section{References}

Abdollahi-Arpanahi R, Morota G and Penagaricano F 2017. Predicting bull fertility using genomic data and biological information. Journal of Dairy Science 100 , 9656-9666. 
Al Naib A, Hanrahan JP, Lonergan P and Fair S 2011. In vitro assessment of sperm from bulls of high and low field fertility. Theriogenology 76, 161-167.

Amann RP and DeJarnette JM 2012. Impact of genomic selection of Al dairy sires on their likely utilization and methods to estimate fertility: a paradigm shift. Theriogenology 77, 795-817.

Aslam MK, Kumaresan A, Sharma VK, Tajmul M, Chhillar S, Chakravarty AK, Manimaran A, Mohanty TK, Srinivasan A and Yadav S 2014. Identification of putative fertility markers in seminal plasma of crossbred bulls through differential proteomics. Theriogenology 82, 1254-1262 e1251.

Beatty RA, Bennett GH, Hall JG, Hancock JL and Stewart DL 1969. An experiment with heterospermic insemination in cattle. Journal of Reproduction and Fertility 19, 491-502.

Boerke A, Dieleman SJ and Gadella BM 2007. A possible role for sperm RNA in early embryo development. Theriogenology 68 (suppl. 1), S147-S155.

Bulek K, Swaidani S, Aronica M and Li X 2010. Epithelium: the interplay between innate and Th2 immunity. Immunology and Cell Biology 88, 257-268.

Cancel AM, Chapman DA and Killian GJ 1997. Osteopontin is the 55-kilodalton fertility-associated protein in Holstein bull seminal plasma. Biology of Reproduction 57, 1293-1301.

Cerri RL, Rutigliano HM, Lima FS, Araujo DB and Santos JE 2009. Effect of source of supplemental selenium on uterine health and embryo quality in high-producing dairy cows. Theriogenology 71, 1127-1137.

Comizzoli P, Marquant-Le Guienne B, Heyman Y and Renard JP 2000. Onset of the first $\mathrm{S}$-phase is determined by a paternal effect during the G1-phase in bovine zygotes. Biology of Reproduction 62, 1677-1684.

D'Amours O, Frenette G, Fortier M, Leclerc P and Sullivan R 2010. Proteomic comparison of detergent-extracted sperm proteins from bulls with different fertility indexes. Reproduction 139, 545-556.

Das PJ, McCarthy F, Vishnoi M, Paria N, Gresham C, Li G, Kachroo P, Sudderth AK, Teague S, Love CC, Varner DD, Chowdhary BP and Raudsepp T 2013. Stallion sperm transcriptome comprises functionally coherent coding and regulatory RNAs as revealed by microarray analysis and RNA-seq. PLoS One 8, e56535.

Den Daas JH, De Jong G, Lansbergen LM and Van Wagtendonk-De Leeuw AM 1998. The relationship between the number of spermatozoa inseminated and the reproductive efficiency of individual dairy bulls. Journal of Dairy Science 81 , 1714-1723.

Dinnyes A, Lonergan P, Fair T, Boland MP and Yang X 1999. Timing of the first cleavage post-insemination affects cryosurvival of in vitro-produced bovine blastocysts. Molecular Reproduction and Development 53, 318-324.

Diskin MG and Sreenan JM 1980. Fertilization and embryonic mortality rates in beef heifers after artificial insemination. Journal of Reproduction and Fertility 59 463-468.

Dogan S, Vargovic P, Oliveira R, Belser LE, Kaya A, Moura A, Sutovsky P, Parrish J, Topper E and Memili E 2015. Sperm protamine-status correlates to the fertility of breeding bulls. Biology of Reproduction 92, 92.

Druart X, Cognie J, Baril G, Clement F, Dacheux JL and Gatti JL 2009. In vivo imaging of in situ motility of fresh and liquid stored ram spermatozoa in the ewe genital tract. Reproduction 138, 45-53.

Druart X, Rickard JP, Mactier S, Kohnke PL, Kershaw-Young CM, Bathgate $R$ Gibb Z, Crossett B, Tsikis G, Labas V, Harichaux G, Grupen CG and de Graaf SP 2013. Proteomic characterization and cross species comparison of mammalian seminal plasma. Journal of Proteomics 91, 13-22.

Evenson D and Jost L 2000. Sperm chromatin structure assay is useful for fertility assessment. Methods in Cell Science 22, 169-189.

Fatima A and Morris DG 2013. MicroRNAs in domestic livestock. Physiological Genomics 45, 685-696.

Fernandez-Fuertes B, Narciandi F, O'Farrelly C, Kelly AK, Fair S, Meade KG and Lonergan P 2016. Cauda epididymis-specific beta-defensin 126 promotes sperm motility but not fertilizing ability in cattle. Biology of Reproduction 95, 122.

Ferreira LS 1972. Factors affecting fertility levels in males as measured by competitive fertilization. PhD thesis, University of Illinois at Urbana-Champaign, Champaign, IL, USA.

Feugang JM, Rodriguez-Osorio N, Kaya A, Wang H, Page G, Ostermeier GC Topper EK and Memili E 2010. Transcriptome analysis of bull spermatozoa: implications for male fertility. Reproductive Biomedicine Online 21, 312-324.

Filho RM, Beletti ME and de Oliveira F 2015. Ultrastructure of bovine sperm chromatin. Microscopy Research and Technique 78, 1117-1120.
Flint AF, Chapman PL and Seidel GE Jr 2003. Fertility assessment through heterospermic insemination of flow-sorted sperm in cattle. Journal of Animal Science 81, 1814-1822.

Gaviraghi A, Deriu F, Soggiu A, Galli A, Bonacina C, Bonizzi L and Roncada P 2010. Proteomics to investigate fertility in bulls. Veterinary Research Communications 34 (suppl. 1), S33-S36.

Govindaraju A, Uzun A, Robertson L, Atli MO, Kaya A, Topper E, Crate EA Padbury J, Perkins A and Memili E 2012. Dynamics of microRNAs in bull spermatozoa. Reproductive Biology and Endocrinology 10, 82.

Hammerstedt RH 1996. Evaluation of sperm quality: Identification of the subfertile male and courses of action. Animal Reproduction Science 42, 77-87. Hung PH and Suarez SS 2012. Alterations to the bull sperm surface proteins that bind sperm to oviductal epithelium. Biology of Reproduction 87, 88.

Jodar M, Selvaraju S, Sendler E, Diamond MP and Krawetz SA 2013. The presence, role and clinical use of spermatozoal RNAs. Human Reproduction Update 19, 604-624.

Johnson GP, English AM, Cronin S, Hoey DA, Meade KG and Fair S 2017. Genomic identification, expression profiling, and functional characterization of CatSper channels in the bovine. Biology of Reproduction 97, 302-312.

Karoui S, Díaz C, González-Marín C, Amenabar M, Serrano M, Ugarte E, Gosálvez J, Roy R, López-Fernández C and Carabaño M 2012. Is sperm DNA fragmentation a good marker for field Al bull fertility? Journal of Animal Science 90, 2437-2449.

Kasimanickam V, Kasimanickam R, Arangasamy A, Saberivand A, Stevenson JS and Kastelic JP 2012. Association between mRNA abundance of functional sperm function proteins and fertility of Holstein bulls. Theriogenology 78, 2007-2019 e2002.

Koppers AJ, Mitchell LA, Wang P, Lin M and Aitken RJ 2011. Phosphoinositide 3-kinase signalling pathway involvement in a truncated apoptotic cascade associated with motility loss and oxidative DNA damage in human spermatozoa. Biochemical Journal 436, 687-698.

Krawetz SA 2005. Paternal contribution: new insights and future challenges. Nature Reviews Genetics 6, 633-642.

Kropp J, Carrillo JA, Namous H, Daniels A, Salih SM, Song J and Khatib H 2017. Male fertility status is associated with DNA methylation signatures in sperm and transcriptomic profiles of bovine preimplantation embryos. BMC Genomics $18,280$.

Kumaresan A, Johannisson A, Al-Essawe EM and Morrell JM 2017. Sperm viability, reactive oxygen species, and DNA fragmentation index combined can discriminate between above-and below-average fertility bulls. Journal of Dairy Science 100, 5824-5836.

Lambert S, Blondin P, Vigneault C, Labrecque R, Dufort I and Sirard MA 2018. Spermatozoa DNA methylation patterns differ due to peripubertal age in bulls. Theriogenology 106, 21-29.

Leahy T and de Graaf SP 2012. Seminal plasma and its effect on ruminant spermatozoa during processing. Reproduction in Domestic Animals 47 (suppl. 4), 207-213.

Lefebvre R, Lo MC and Suarez SS 1997. Bovine sperm binding to oviductal epithelium involves fucose recognition. Biology of Reproduction 56, 1198-1204. Legare C, Akintayo A, Blondin P, Calvo E and Sullivan R 2017. Impact of male fertility status on the transcriptome of the bovine epididymis. Molecular Human Reproduction 23, 355-369.

Lessard C, Siqueira LG, D'Amours 0, Sullivan R, Leclerc P and Palmer C 2011. Infertility in a beef bull due to a failure in the capacitation process. Theriogenology 76, 891-899.

Li JC and Funahashi H 2010. Effect of blood serum, caffeine and heparin on in vitro phagocytosis of frozen-thawed bull sperm by neutrophils derived from the peripheral blood of cows. Theriogenology 74, 691-698.

Lonergan P, Khatir H, Piumi F, Rieger D, Humblot P and Boland MP 1999. Effect of time interval from insemination to first cleavage on the developmental characteristics, sex ratio and pregnancy rate after transfer of bovine embryos. Journal of Reproduction and Fertility 117, 159-167.

Lyons A, Narciandi F, Donnellan E, Romero-Aguirregomezcorta J, O'Farrelly C, Lonergan P, Meade KG and Fair S 2018. Recombinant beta-defensin 126 promotes bull sperm binding to bovine oviductal epithelia. Reproduction Fertility and Development (In Press).

Macmillan KL and Watson JD 1975. Fertility differences between groups of sires relative to the stage of oestrus at the time of insemination. Animal Science 21 243-249. 
Fair and Lonergan

Macmillan KL and Curnow RJ 1977. Factors influencing A.B conception rates: XI. Variation in sire fertility. New Zealand Journal of Experimental Agriculture 5, 279-285.

Marey MA, Liu J, Kowsar R, Haneda S, Matsui M, Sasaki M, Takashi S, Hayakawa H, Wijayagunawardane MP, Hussein FM and Miyamoto A 2014. Bovine oviduct epithelial cells downregulate phagocytosis of sperm by neutrophils: prostaglandin E2 as a major physiological regulator. Reproduction 147, 211-219.

Marey MA, Yousef MS, Liu J, Morita K, Sasaki M, Hayakawa H, Shimizu T, Elshahawy II and Miyamoto A 2016. Endothelin-1 downregulates sperm phagocytosis by neutrophils in vitro: A physiological implication in bovine oviduct immunity. Journal of Reproduction and Development 62, 151-157.

Miki $\mathrm{K}$ and Clapham DE 2013. Rheotaxis guides mammalian sperm. Current Biology 23, 443-452.

Murphy C, Holden SA, Murphy EM, Cromie AR, Lonergan P and Fair S 2015. The impact of storage temperature and sperm number on the fertility of liquid-stored bull semen. Reproduction Fertility and Development (In Press).

Murphy EM, Eivers B, O'Meara CM, Lonergan P and Fair S 2017. Effect of storage temperature, nitrogen gassing and sperm concentration on the in vitro semen quality and in vivo fertility of liquid bull semen stored in INRA96. Theriogenology 108, 223-228.

Nagy S, Johannisson A, Wahlsten T, ljas R, Andersson M and RodriguezMartinez H 2013. Sperm chromatin structure and sperm morphology: their association with fertility in Al-dairy Ayrshire sires. Theriogenology 79, 1153-1161.

Narciandi F, Fernandez-Fuertes B, Khairulzaman I, Jahns $H$, King D, Finlay EK Mok KH, Fair S, Lonergan P, Farrelly CO and Meade KG 2016. Sperm-coating beta-defensin 126 is a dissociation-resistant dimer produced by epididyma epithelium in the bovine reproductive tract. Biology of Reproduction 95, 121.

Narciandi F, Lloyd AT, Chapwanya A, C OF and Meade KG 2011. Reproductive tissue-specific expression profiling and genetic variation across a 19 gene bovine beta-defensin cluster. Immunogenetics 63, 641-651.

Ortega MS, Moraes JGN, Patterson DJ, Smith MF, Poock S and Spencer TE 2017.

Effect of sire conception rate on pregnancy establishment in dairy cattle. Proceedings of the $50^{\text {th }}$ Society for the Study of Reproduction, 13-16 July, Washington, DC, USA, p. 100.

Overstreet JW and Adams CE 1971. Mechanisms of selective fertilization in the rabbit: sperm transport and viability. Journal of Reproduction and Fertility 26 , 219-231.

Park YJ, Kwon WS, Oh SA and Pang MG 2012. Fertility-related proteomic profiling bull spermatozoa separated by percoll. Journal of Proteome Research 11, 4162-4168.

Puglisi R, Gaspa G, Balduzzi D, Severgnini A, Vanni R, Macciotta N and Galli A 2016. Genomewide analysis of bull sperm quality and fertility traits. Reproduction in Domestic Animals 51, 840-843.

Rahman MS, Kwon WS and Pang MG 2017. Prediction of male fertility using capacitation-associated proteins in spermatozoa. Molecular Reproduction and Development 84, 749-759.

Rodriguez-Martinez $\mathrm{H}$ and Barth A 2007. In vitro evaluation of sperm quality related to in vivo function and fertility. Society of Reproduction and Fertility 64 (suppl.), 39-54.

Rossato M, Galeazzi C, Ferigo M and Foresta C 2004. Antisperm antibodies modify plasma membrane functional integrity and inhibit osmosensitive calcium influx in human sperm. Human Reproduction 19, 1816-1820.

Sakkas D, Moffatt O, Manicardi GC, Mariethoz E, Tarozzi N and Bizzaro D 2002. Nature of DNA damage in ejaculated human spermatozoa and the possible involvement of apoptosis. Biology of Reproduction 66, 1061-1067.

Sartori R, Bastos MR and Wiltbank MC 2010. Factors affecting fertilisation and early embryo quality in single- and superovulated dairy cattle. Reproduction Fertility and Development 22, 151-158.

Sartori R, Sartor-Bergfelt R, Mertens SA, Guenther JN, Parrish JJ and Wiltbank MC 2002. Fertilization and early embryonic development in heifers and lactating cows in summer and lactating and dry cows in winter. Journal of Dairy Science 85, 2803-2812.

Schjenken JE and Robertson SA 2014. Seminal fluid and immune adaptation for pregnancy-comparative biology in mammalian species. Reproduction in Domestic Animals 49 (suppl. 3), 27-36.

Schuberth HJ, Taylor U, Zerbe H, Waberski D, Hunter R and Rath D 2008 Immunological responses to semen in the female genital tract. Theriogenology 70, 1174-1181.
Sellem E, Broekhuijse ML, Chevrier L, Camugli S, Schmitt E, Schibler L and Koenen EP 2015. Use of combinations of in vitro quality assessments to predic fertility of bovine semen. Theriogenology 84, 1447-1454 e1445.

Singh RK, Kumaresan A, Chhillar S, Rajak SK, Tripathi UK, Nayak S, Datta T, Mohanty T and Malhotra R 2016. Identification of suitable combinations of in vitro sperm-function test for the prediction of fertility in buffalo bull. Theriogenology 86, 2263-2271. e2261.

Somashekar L, Selvaraju S, Parthipan S, Patil SK, Binsila BK, Venkataswamy MM, Karthik Bhat $S$ and Ravindra JP 2017. Comparative sperm protein profiling in bulls differing in fertility and identification of phosphatidylethanolamine-binding protein 4, a potential fertility marker. Andrology 5, 1032-1051.

Somashekar L, Selvaraju S, Parthipan S and Ravindra JP 2015. Profiling of sperm proteins and association of sperm PDC-109 with bull fertility. Systems Biology in Reproductive Medicine 61, 376-387.

Sreenan JM and Diskin MG 1986. The extent and timing of embryonic mortality in the cow. In Embryonic mortality in farm animals (ed. JM Sreenan and MG Diskin), pp 1-11. Springer, Dordrecht, The Netherlands.

Takeda K, Uchiyama K, Kinukawa M, Tagami T, Kaneda M and Watanabe S 2015. Evaluation of sperm DNA damage in bulls by TUNEL assay as a parameter of semen quality. Journal of Reproduction and Development 61, 185-190.

Tollner TL, Bevins CL and Cherr GN 2012. Multifunctional glycoprotein DEFB126-a curious story of defensin-clad spermatozoa. Nature Reviews Urology 9, 365-375.

Tollner TL, Venners SA, Hollox EJ, Yudin Al, Liu X, Tang G, Xing H, Kays RJ, Lau T, Overstreet JW, Xu X, Bevins CL and Cherr GN 2011. A Common Mutation in the Defensin DEFB126 Causes Impaired Sperm Function and Subfertility. Science Translational Medicine 3, 92ra65.

Tollner TL, Yudin Al, Tarantal AF, Treece CA, Overstreet JW and Cherr GN 2008 Beta-defensin 126 on the surface of macaque sperm mediates attachment of sperm to oviductal epithelia. Biology of Reproduction 78, 400-412.

Tscherner A, Gilchrist G, Smith N, Blondin P, Gillis D and LaMarre J 2014. MicroRNA-34 family expression in bovine gametes and preimplantation embryos. Reproductive Biology and Endocrinology 12, 85.

Utt MD 2016. Prediction of bull fertility. Animal Reproduction Science 169, 37-44.

Vishwanath R and Shannon P 2000. Storage of bovine semen in liquid and frozen state. Animal Reproduction Science 62, 23-53.

Ward F, Enright B, Rizos D, Boland M and Lonergan P 2002. Optimization of in vitro bovine embryo production: effect of duration of maturation, length of gamete co-incubation, sperm concentration and sire. Theriogenology 57, 2105-2117.

Ward F, Rizos D, Corridan D, Quinn K, Boland M and Lonergan P 2001. Paternal influence on the time of first embryonic cleavage post insemination and the implications for subsequent bovine embryo development in vitro and fertility in vivo. Molecular Reproduction and Development 60, 47-55.

Whiston R, Finlay EK, McCabe MS, Cormican P, Flynn P, Cromie A, Hansen PJ, Lyons A, Fair S, Lonergan P, O' Farrelly C and Meade KG 2017. A dual targeted betadefensin and exome sequencing approach to identify, validate and functionally characterise genes associated with bull fertility. Scientific Reports 7, 12287.

Xu ZZ 2017. Effect of time of artificial insemination after oestrus detection on non-return rate of lactating dairy cows on pasture. New Zealand Veterinary Journal 65, 119-123.

Yousef MS, Marey MA, Hambruch N, Hayakawa $H$, Shimizu T, Hussien HA Abdel-Razek AK, Pfarrer C and Miyamoto A 2016. Sperm binding to oviduct epithelial cells enhances TGFB1 and IL10 expressions in epithelial cells as well as neutrophils in vitro: prostaglandin E2 As a main regulator of anti-inflammatory response in the bovine oviduct. PLoS One 11, e0162309.

Yudin Al, Generao SE, Tollner TL, Treece CA, Overstreet JW and Cherr GN 2005. Beta-defensin 126 on the cell surface protects sperm from immunorecognition and binding of anti-sperm antibodies. Biology of Reproduction 73, 1243-1252.

Zhou YS, Webb S, Lettice L, Tardif S, Kilanowski F, Tyrrell C, Macpherson H Semple F, Tennant P, Baker T, Hart A, Devenney P, Perry P, Davey T, Barran P, Barratt $C L$ and Dorin JR 2013. Partial deletion of chromosome 8 beta-defensin cluster confers sperm dysfunction and infertility in male mice. PLoS Genetics 9, e1003826.

Zodinsanga V, Cheema RS and Mavi PS 2015. Relationship of naturally occurring antisperm antibodies in blood serum and seminal plasma of cattle bulls with sperm function and fertility tests. Open Journal of Animal Sciences 5, 114-123. 